\title{
Free Deformable Nanofibers Enhanced Tribo-Sensors for Sleep and Tremor Monitoring
}

\author{
Ran Cao ${ }^{\dagger, \downarrow}$, Shuyu Zhao ${ }^{\dagger, \downarrow}$, and Congju $\mathbf{L i}^{*, \S, \|}$ \\ ${ }^{\dagger}$ Beijing Institute of Nanoenergy and Nanosystems, Chinese Academy of Sciences, Beijing \\ 100083, China \\ School of Nanoscience and Technology, University of Chinese Academy of Sciences, Beijing \\ 100049, China \\ ${ }^{\S}$ School of Energy and Environmental Engineering, University of Science and Technology Beijing, \\ Beijing 100083, China \\ "Beijing Key Laboratory of Resource-oriented Treatment of Industrial pollutants, Beijing 100083, \\ China
}

E-mail: congjuli@126.com
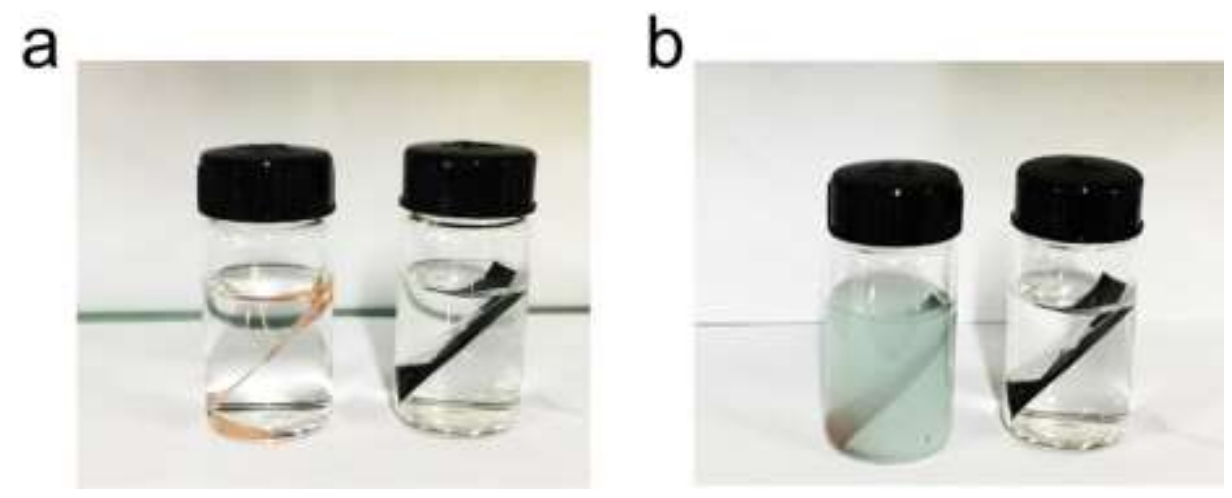

Figure S1. The optical photographs of the stability of the CNT ink in salt solution. The solutions of the $\mathrm{NaCl}(15 \mathrm{wt} \%)$ before a) and after a week b) with copper strip 
and CNT ink strip immersed into it, respectively.

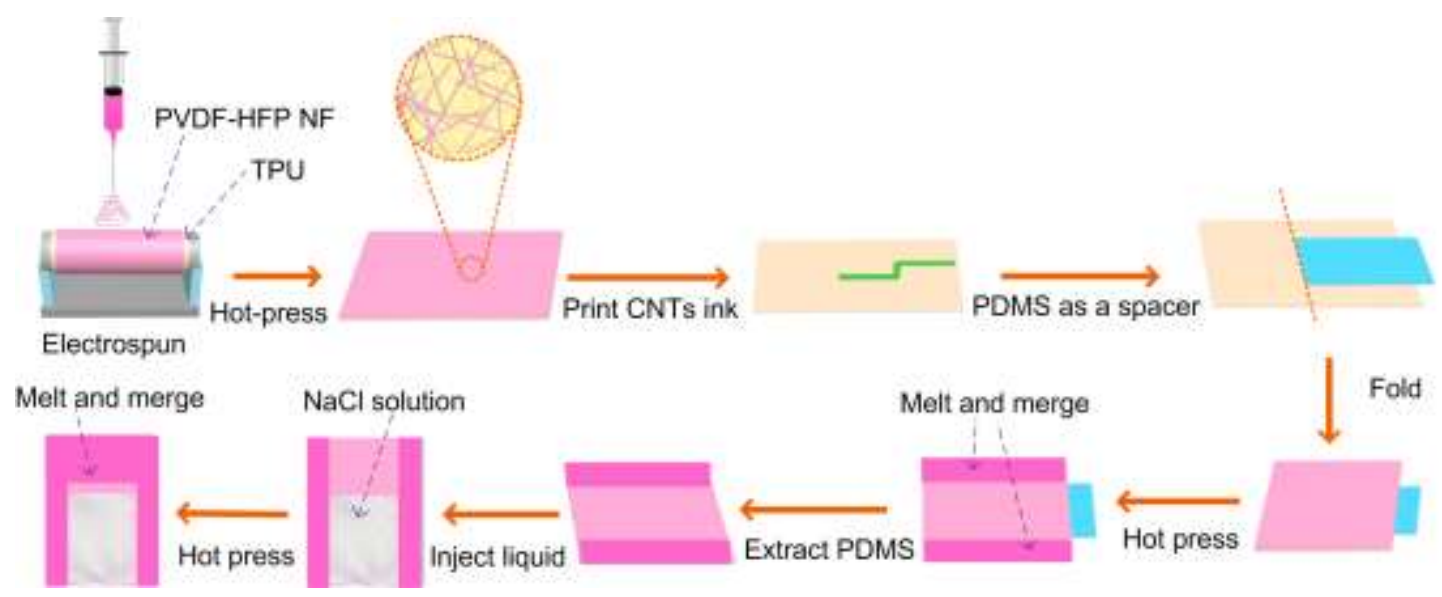

Figure S2 The scheme of the fabrication process of the FDTS.

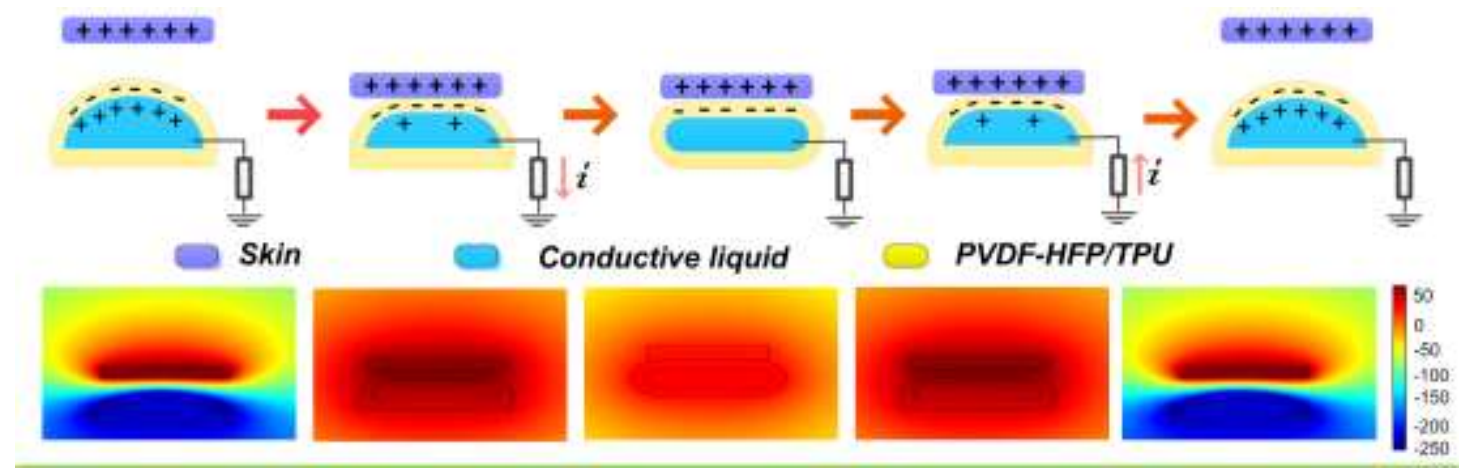

Figure S3 The potential distribution of the FDTS during one working period (simulated by COMSOL).

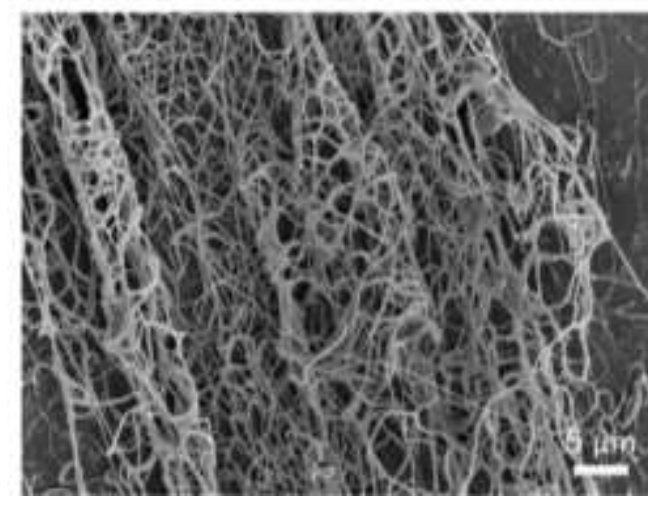


Figure S4 The SEM images of the surface of the composite membrane. The PVDF-HFP layer (electrospun for $40 \mathrm{~min}$ ) separated with the substrate layer.

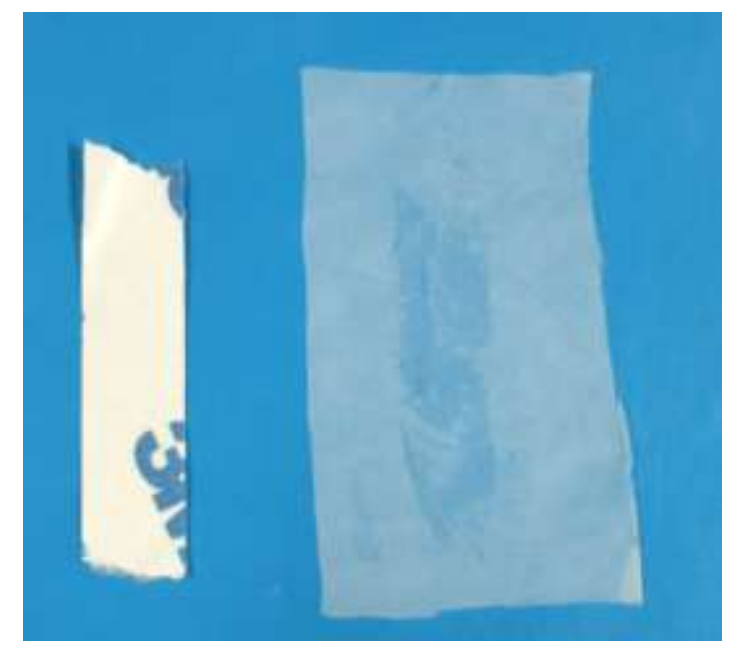

Figure S5 The optical photo of the surface of the PVDF-HFP / TPU composite after being tested by $3 \mathrm{M}$ tape.

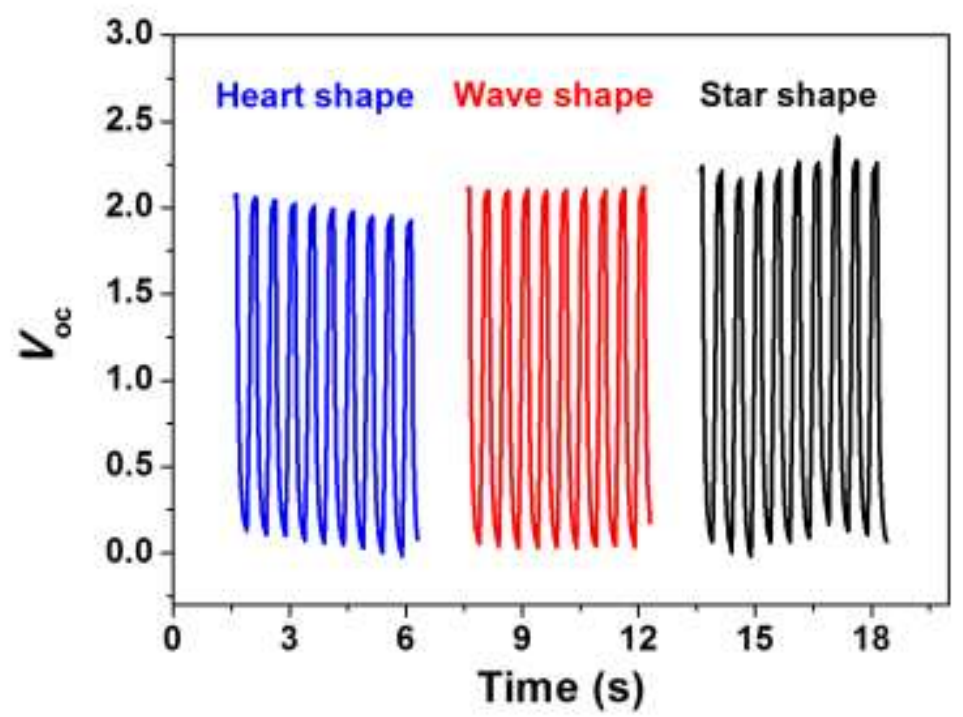

Figure S6 The $V_{\text {oc }}$ of the FDTS under different deformation. 


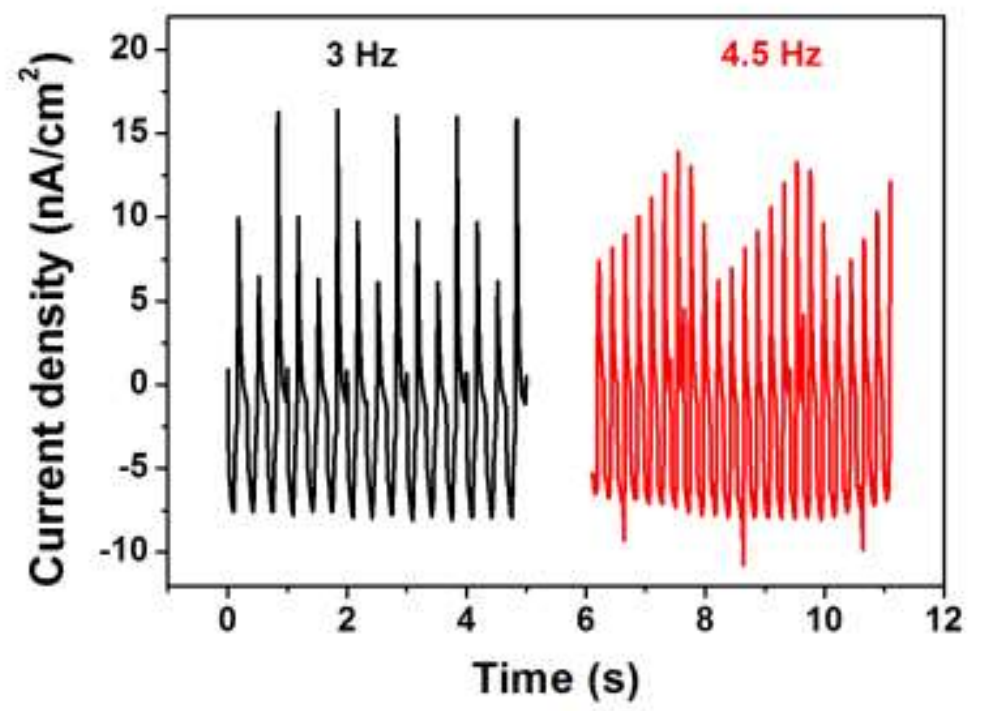

Figure S7 The current density signals of the FDTS under $3 \mathrm{~Hz}$ and $4.5 \mathrm{~Hz}$.

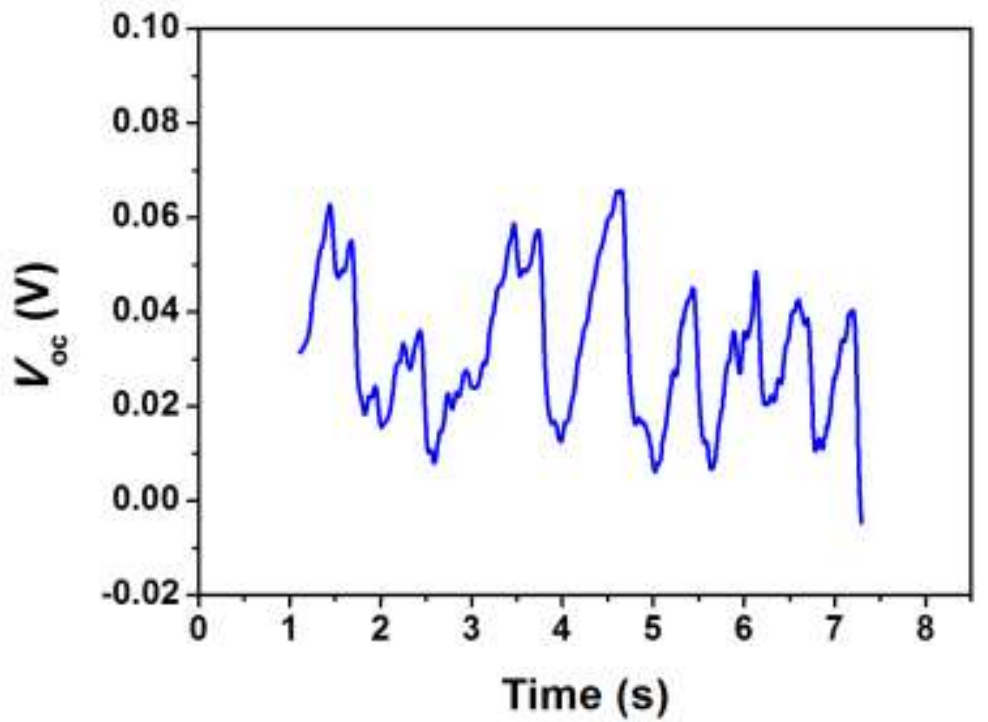

Figure S8 The $V_{\text {oc }}$ signals of the FDTS during monitoring eye wink with small magnitude. 


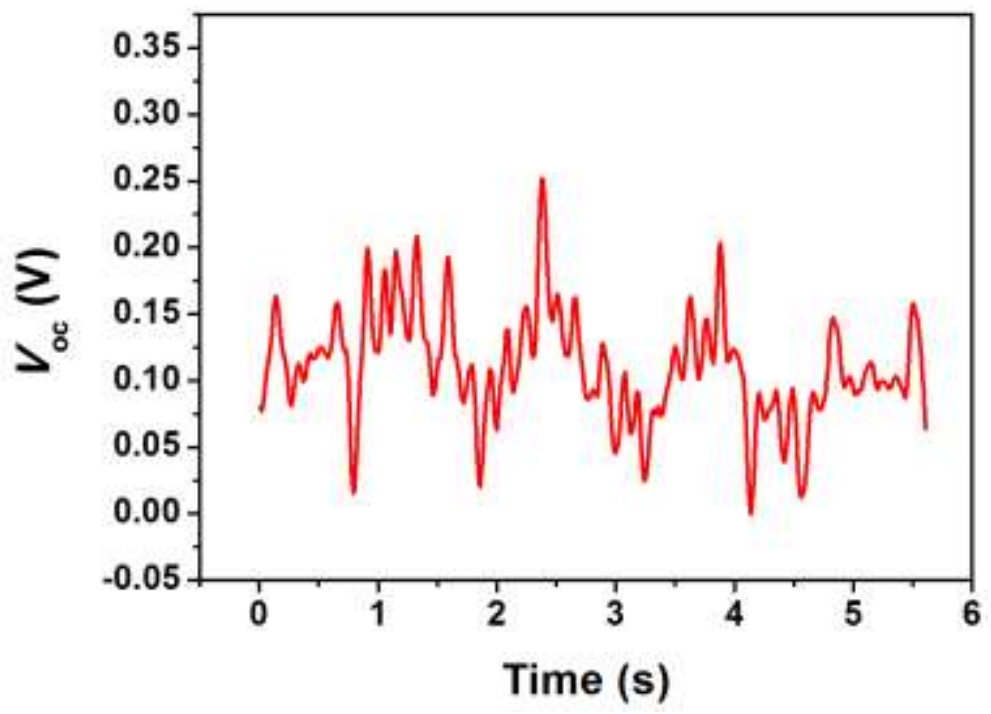

Figure S9 The $V_{\mathrm{oc}}$ signals of the FDTS during monitoring tremor with small magnitude and high frequency.

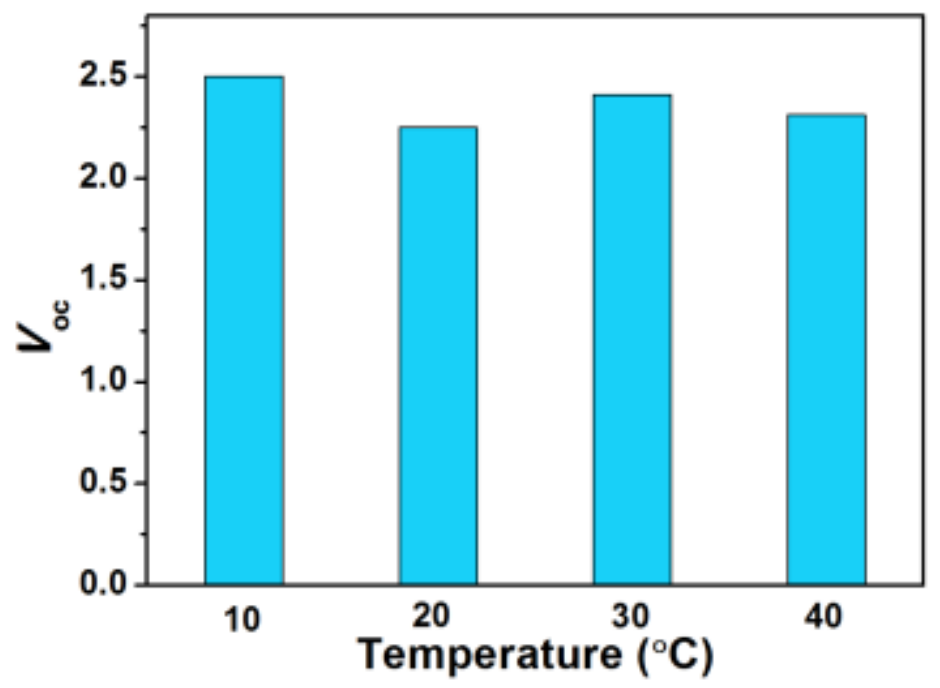

Figure S10 The output of the FDTS under temperature range from $10{ }^{\circ} \mathrm{C}$ to $40^{\circ} \mathrm{C}$. 


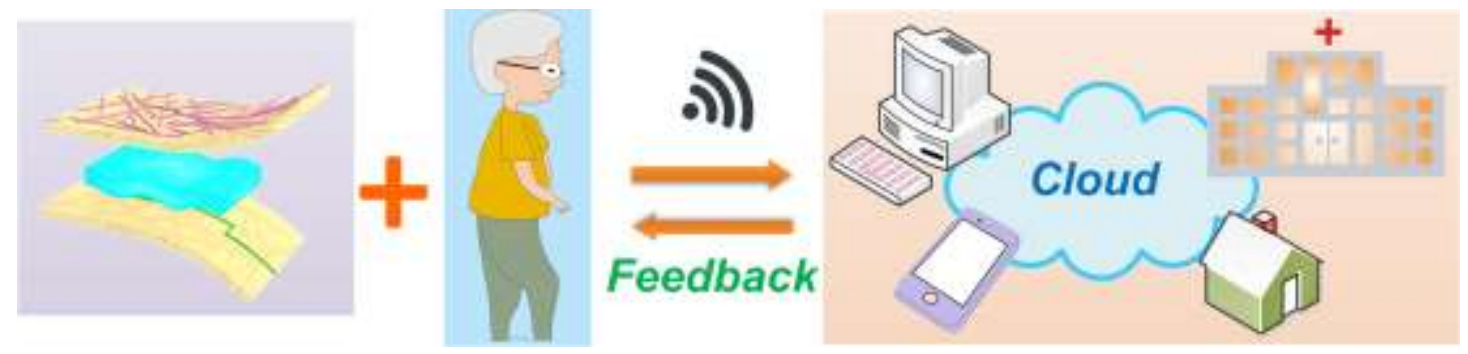

Figure S11 The scheme diagram of the FDTS involved in a smart health monitoring system for the aged.

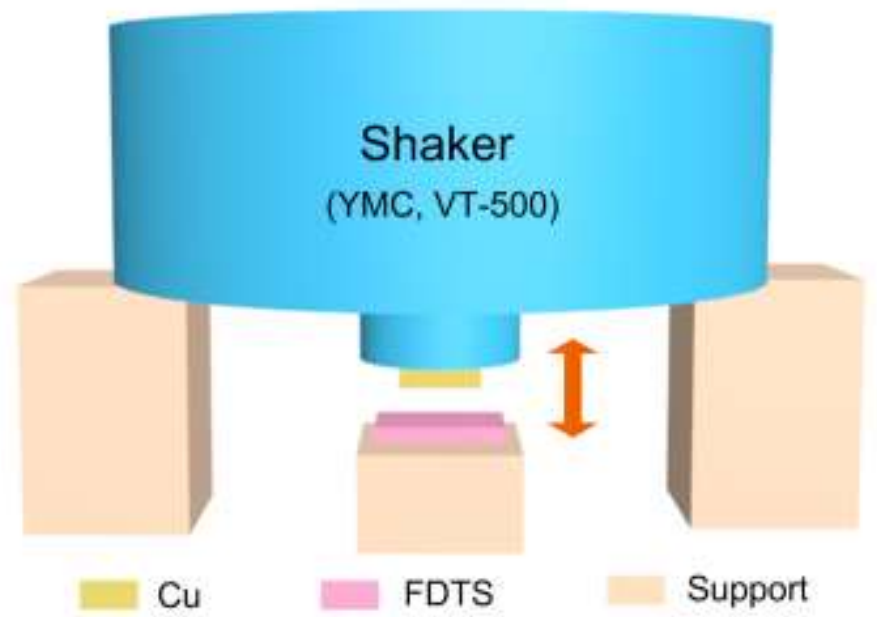

Figure S12 The scheme diagram of the characterization (electrical performance) setup for FDTS.

Table S1 The electric conductivity of the conductive liquid with different concentration of $\mathrm{NaCl}$.

\begin{tabular}{llccc}
\hline Concentration & $\mathbf{0 \%}$ & $\mathbf{5 \%}$ & $\mathbf{1 5 \%}$ & $\mathbf{2 5 \%}$ \\
of $\mathrm{NaCl}(\boldsymbol{\%})$ & & & & \\
\hline Electric & 2.49 & 74.9 & 180.8 & 239.24 \\
conductivity & & & \\
\hline$(\mu \mathrm{s} / \mathrm{cm})$ & & & \\
\hline
\end{tabular}


